\title{
Salmonella inhibits tumor angiogenesis by downregulation of vascular endothelial growth factor
}

\author{
Dom-Gene Tu1,2,3, Wen-Wei Chang ${ }^{4,5}$, Song-Tao Lin', Chun-Yu Kuo ${ }^{7}$, Yu-Tzu Tsao ${ }^{8}$ \\ and Che-Hsin Lee (6,7,9 $^{6}$ \\ ${ }^{1}$ Department of Nuclear Medicine, Ditmanson Medical Foundation, Chia-Yi Christian Hospital, Chia-Yi, Taiwan \\ ${ }^{2}$ Department of Food Science and Technology, Chia Nan University of Pharmacy and Science, Tainan, Taiwan \\ ${ }^{3}$ College of Health Sciences, Chang Jung Christian University, Tainan, Taiwan \\ ${ }^{4}$ Department of Biomedical Sciences, College of Medical Science and Technology, Chung Shan Medical University, Taichung, \\ Taiwan \\ ${ }^{5}$ Department of Medical Research, Chung Shan Medical University Hospital, Taichung, Taiwan \\ ${ }^{6}$ Department of Microbiology, School of Medicine, China Medical University, Taichung, Taiwan \\ ${ }^{7}$ Graduate Institute of Basic Medical Science, School of Medicine, China Medical University, Taichung, Taiwan \\ 8 Department of Medicine, Taoyuan General Hospital, Taoyuan, Taiwan \\ ${ }^{9}$ Department of Biological Sciences, National Sun Yat-sen University, Kaohsiung, Taiwan \\ Correspondence to: Che-Hsin Lee, email: chlee@mail.cmu.edu.tw \\ Keywords: Salmonella, tumor-targeting, angiogenesis, hypoxia-inducible factor-1a, vascular endothelial growth factor \\ Received: August 10, $2015 \quad$ Accepted: January 19, $2016 \quad$ Published: January 27, 2016
}

\section{ABSTRACT}

Salmonella is a Gram-negative, facultative anaerobe that is a common cause of host intestinal infections. Salmonella grows under aerobic and anaerobic conditions, and it has been proven capable of inhibiting tumor growth. However, the molecular mechanism by which Salmonella inhibits tumor growth is still unclear. Angiogenesis plays an important role in the development and progression of tumors. We investigated the antitumor effect of Salmonella in a syngeneic murine tumor model. Hypoxia-inducible factor-1 (HIF-1)a plays a significant role in tumor angiogenesis. We examined the molecular mechanism by which Salmonella regulated vascular endothelial growth factor (VEGF), which is an important angiogenic factor. The expression of VEGF in tumor cells was decreased by treatment with Salmonella. The conditioned medium from Salmonella-treated cells inhibited the proliferation of endothelial cells. Salmonella inhibited the expression of HIF-1a as well as downregulated its upstream signal mediator protein kinase B (AKT). Salmonella significantly inhibited tumor growth in vivo, and immunohistochemical studies of the tumors revealed decreased intratumoral microvessel density. These results suggest that Salmonella therapy, which exerts anti-angiogenic activities, represents a promising strategy for the treatment of tumors.

\section{INTRODUCTION}

Attenuated Salmonella has been demonstrated to inhibit tumor growth in a broad range of human and mouse tumors [1-9]. There are many advantages of using Salmonella for cancer treatment, including tumortargeting, immunostimulation and low cost [10, 11]. Salmonella stimulates host immunity and reduces tumor growth [12, 13]. Additionally, Salmonella was shown to replicate much more in tumors than in normal tissue and to target to the hypoxic regions in tumors $[14,15]$.

A hypoxic microenvironment is a hallmark of many solid tumors. Hypoxia is also associated with a more malignant phenotype, affecting genomic stability, apoptosis, autophagy, angiogenesis and metastasis [16]. Induction of angiogenesis plays an important role in the development and progression of most tumors. Targeting angiogenesis to inhibit tumor growth is one of the promising therapeutic approaches for tumor treatment [17]. Interestingly, a previous study revealed that mice treated with Salmonella alone, compared with those 
treated with Phosphate-buffered saline (PBS), showed slightly reduced intratumoral microvessel density [15]. To date, a possible interaction of Salmonella with tumor cells has not been examined. Herein, we propose a role for Salmonella in controlling tumor growth by reducing vascular endothelial growth factor (VEGF) expression.

\section{RESULTS}

\section{Salmonella reduced the proliferation of endothelial cells}

Previous studies found that the conditioned medium from Salmonella-infected tumor cells moderately inhibited the proliferation of endothelial cells [14]. Herein, the proliferation of endothelial cells was measured in the conditioned medium of tumor cells treated with Salmonella. The results show that the amounts of endothelial cells were lowest when endothelial cells were cultured with the conditioned medium of B16F10 cells treated with the highest dose of Salmonella (multiplicity of infection $(\mathrm{MOI})=10)$ compared with the control group (Figure 1A). Similar results were observed in 4T1 cells treated with Salmonella (Figure 1B). Collectively, these results suggest that Salmonella may decrease the proliferation of endothelial cells through reducing growth factors produced by tumor cells. The above findings prompted us to further explore the detailed mechanism underlying the anti-angiogenic effects of Salmonella in tumors.

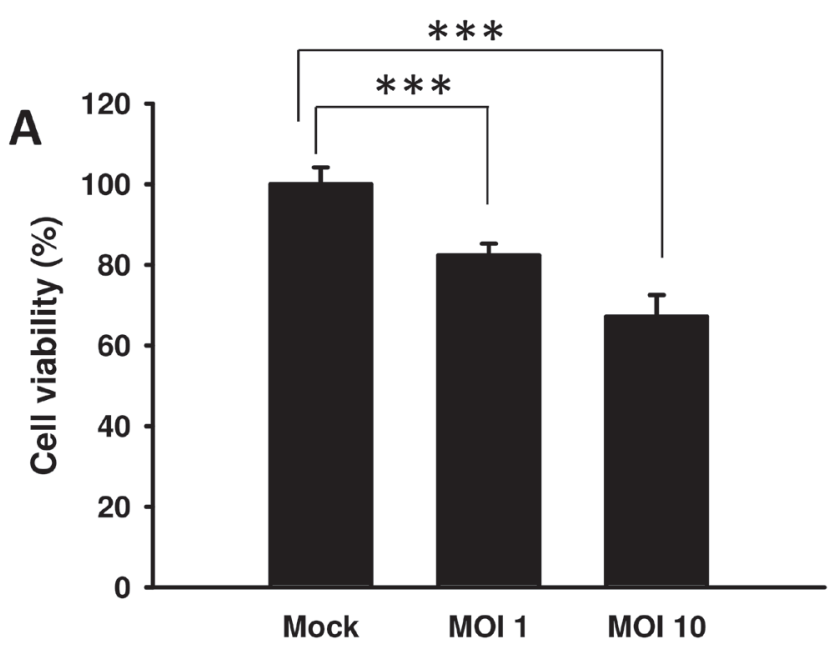

Salmonella reduced HIF-1a expression through the phospho-protein kinase B (P-AKT)/ phosphomammalian target of the rapamycin (P-mTOR) pathway

Vascular endothelial growth factor (VEGF), a major growth factor, can induce angiogenesis during tumor growth. As shown in Figure 2A and 2B, the protein levels of VEGF were dramatically decreased in Salmonellatreated tumor cells. Because HIF- $1 \alpha$ can induce VEGF expression, the expression of HIF-1 $\alpha$ in tumors was measured. The expression of HIF- $1 \alpha$ was decreased in Salmonella-treated tumor cells (B16F10 and 4T1). The extent of hypoxia responsiveness of the hypoxiaresponse element (HRE) reporter assay in two tumor cell lines varied, ranging from 0.91-0.76-fold compared with control cells (Figure 2C and 2D). The levels of hypoxia responsiveness were lower in Salmonella-treated tumor cells (B16F10 and 4T1) than those in mock-infected cells. Because Salmonella can influence HIF-1 $\alpha$ protein expression and thereby abrogate HIF-1 $\alpha$-mediated transcriptional activity in tumor cells, we wanted to identify the signaling pathway affected by Salmonella during tumor angiogenesis. Some studies demonstrated that the protein kinase B (AKT)/ mammalian target of rapamycin (mTOR) signaling pathway can promote HIF-1 $\alpha$ protein synthesis through phosphorylation of protein translational regulators, such as ribosomal p70S6 kinase (p70S6K1) [18, 19]. As expected, the elevated phosphorylation of AKT, mTOR, and p70S6K in B16F10 and 4T1 cells was significantly diminished by Salmonella treatment (Figure 2A and 2B). Taken together, treatment with Salmonella decreased the phosphorylation of AKT, mTOR and p70S6K in a dose-dependent manner, indicating downregulation of the AKT/mTOR/p70S6K/

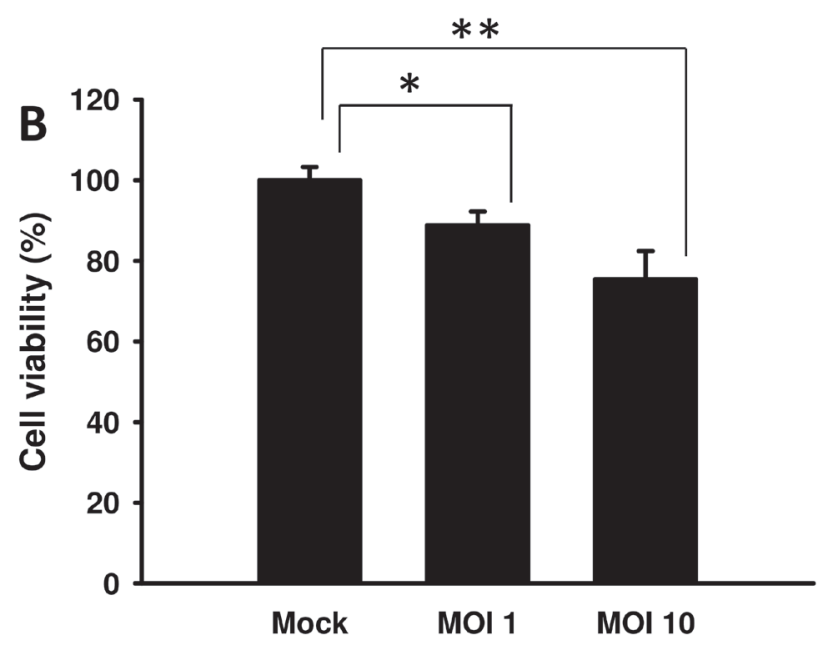

Figure 1: The effect of medium conditioned with Salmonella (S.C.) -treated tumor cells on endothelial cells. The conditioned medium of Salmonella-treated B16F10 A. and 4T1 B. cells reduced the proliferation of endothelial cells. The HEMC-1 cells treated with the conditioned medium of Salmonella-treated tumor cells were examined via proliferation activity. Cell viability was assessed by the WST1 assay. Data shown are the mean $\pm \operatorname{SD}(n=4)$. Multiplicity of infection (MOI). ${ }^{*} p<0.05 ; * * p<0.01 ; * * * p<0.001$. 
A

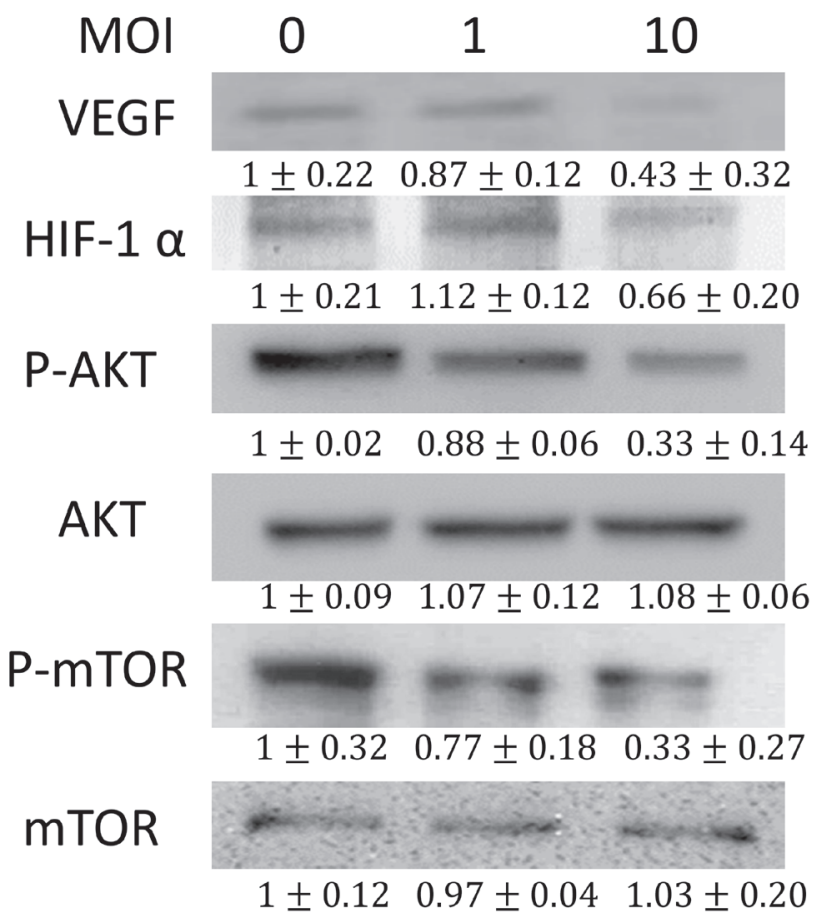

B

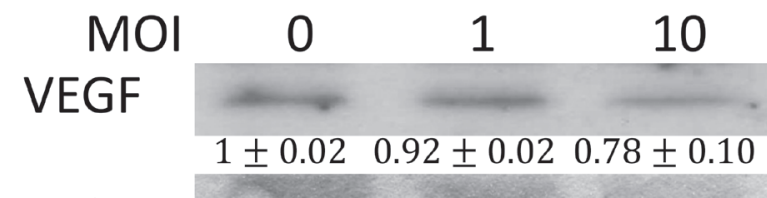

HIF-1 $\alpha$

$1 \pm 0.23 \quad 0.61 \pm 0.22 \quad 0.48 \pm 0.16$

P-AKT

AKT

$1 \pm 0.04 \quad 0.84 \pm 0.060 .21 \pm 0.10$

$1 \pm 0.051 .04 \pm 0.261 .08 \pm 0.11$

\section{P-mTOR}

mTOR

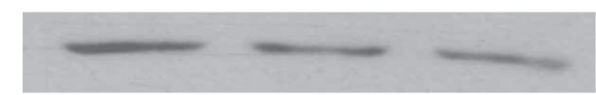

$1 \pm 0.01 \quad 0.91 \pm 0.06 \quad 0.81 \pm 0.03$

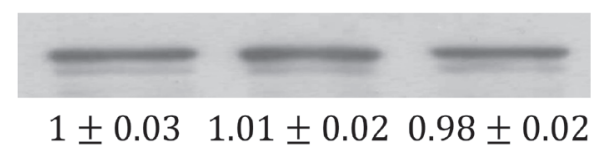

P-p70s6K

$1 \pm 0.09 \quad 0.90 \pm 0.10 \quad 0.58 \pm 0.17$

p70s6K

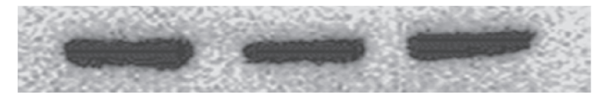

$1 \pm 0.03 \quad 1.01 \pm 0.021 .08 \pm 0.11$
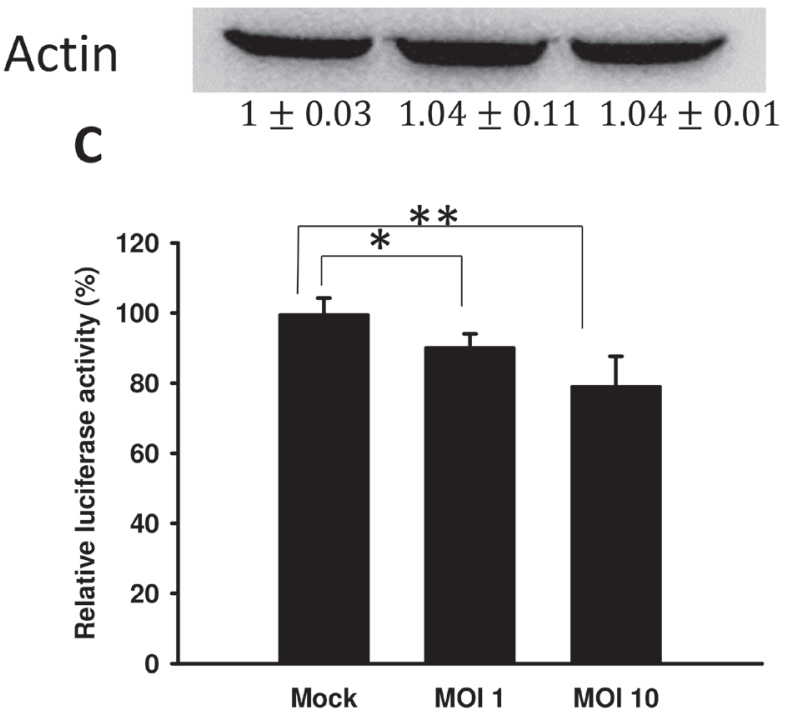

P-p70s6K

p70s6K

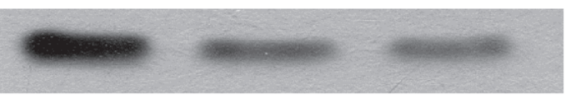

$1 \pm 0.13 \quad 0.79 \pm 0.02 \quad 0.70 \pm 0.11$

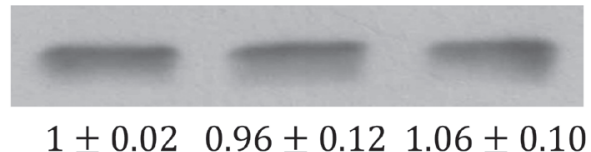

Actin

$1 \pm 0.011 .01 \pm 0.021 .08 \pm 0.11$

D

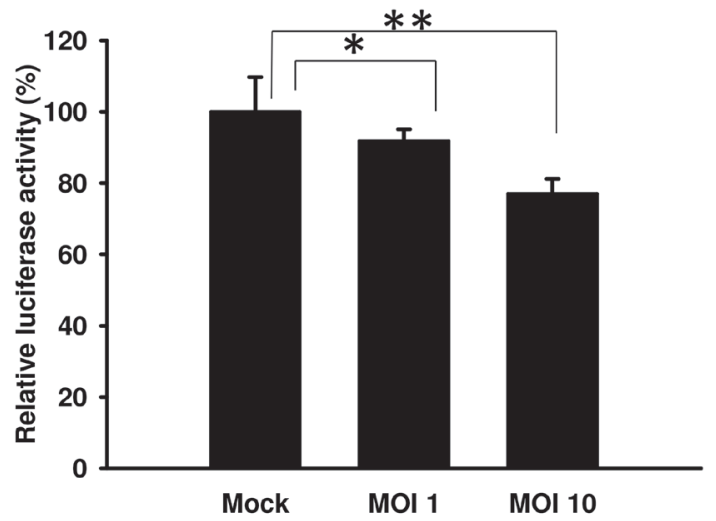

Figure 2: Salmonella (S.C.) regulated the expression of HIF-1 $\alpha$, vascular endothelial growth factor (VEGF), and AKT/mTOR signaling pathway components. The expression of VEGF, HIF-1 $\alpha$, AKT, mTOR, and p70s6K was measured in B16F10 A. and 4T1 B. cells by Western blot analysis. $\beta$-actin expression served as a loading control and as an indicator of total protein levels. Inserted values indicate relative protein expression in comparison with $\beta$-actin $(\mathrm{MOI}=0)$. Each experiment was repeated three times with similar results. The data shown are the mean $\pm \mathrm{SD}(n=3)$. Salmonella-treated B16F10 C. and 4T1 D. cells were cotransfected with pCLNCX-6× HRELuc and pTCYLacZ plasmids. At $6 \mathrm{~h}$ post-transfection, their luciferase activities were determined and normalized with $\beta$-gal activity. Multiplicity of infection (MOI). Data shown are the mean $\pm \operatorname{SD}(n=4)$. $* p<0.05 ; * * p<0.01$. 


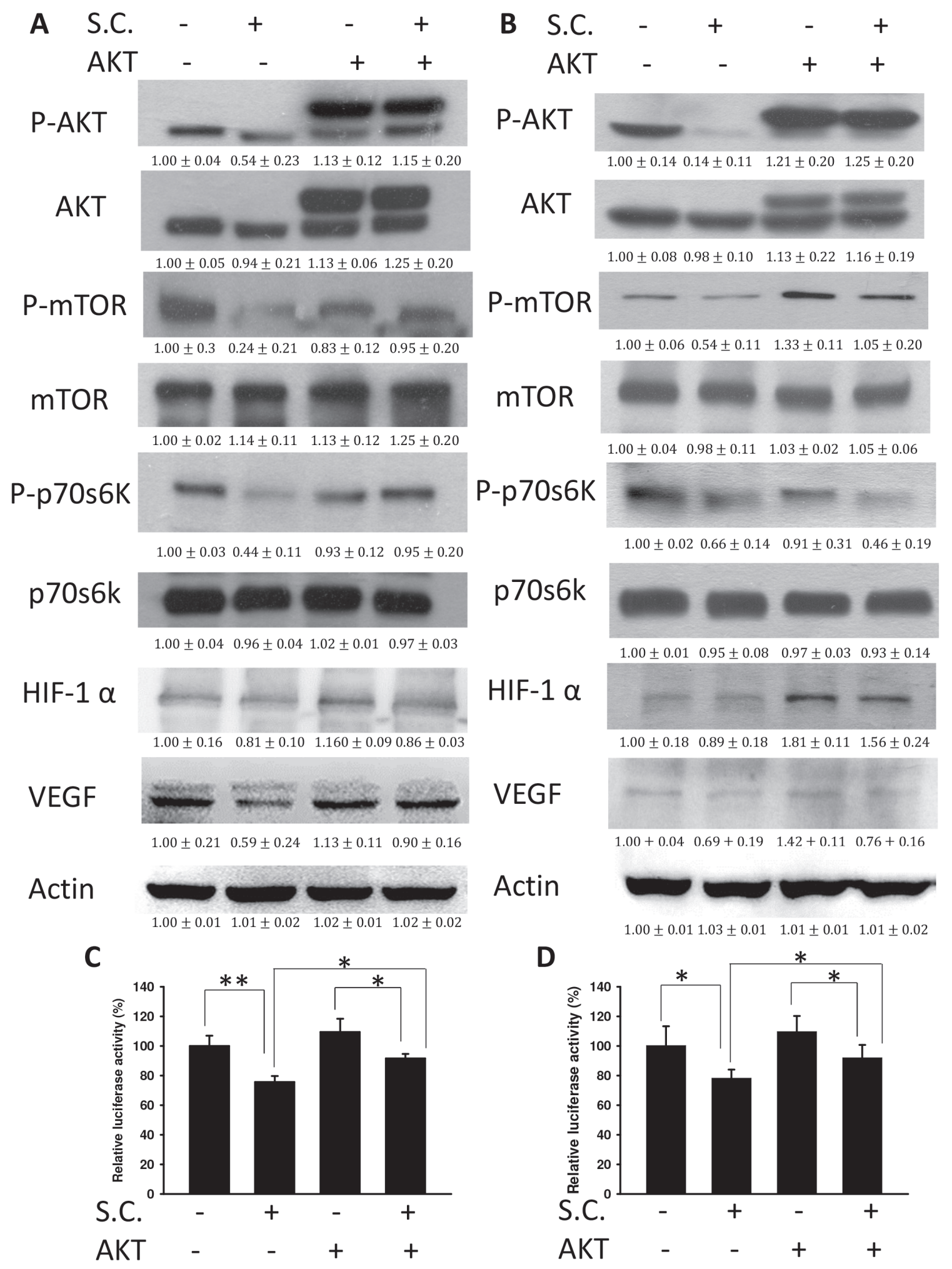

Figure 3: Constitutively active AKT reduced the effects of Salmonella (S.C.). The B16F10 and 4T1 cells were transfected with a constitutively active AKT plasmid $(5 \mu \mathrm{g})$ at $37^{\circ} \mathrm{C}$ for 16 hours prior to infection with Salmonella $(\mathrm{MOI}=10)$ for $4 \mathrm{~h}$. The expression of VEGF, HIF-1 $\alpha$, AKT, mTOR, p70s6K protein in B16F10 A. and 4T1 cells B. was determined. $\beta$-actin expression served as a loading control and as an indicator of total protein levels. Inserted values indicate relative protein expression in comparison with $\beta$-actin $(\mathrm{MOI}=0)$. Each experiment was repeated three times with similar results. Data shown are the mean \pm SD $(n=3)$. Salmonella-treated B16F10 C. and 4T1 D. cells were cotransfected with pCLNCX-6× HRELuc and pTCYLacZ plasmids. At $6 \mathrm{~h}$ post-transfection, their luciferase activities were determined and normalized with $\beta$-gal activity. Multiplicity of infection (MOI). The data shown are the mean $\pm \operatorname{SD}(n=4)$. * $p<0.05 ; * *$ $p<0.01$. 
HIF-1 $\alpha /$ VEGF pathway in tumor cells treated with Salmonella. These results indicated that reduction of VEGF expression by Salmonella in tumor cells was associated with inhibition of the AKT/mTOR/p70S6K pathway.

\section{Salmonella reduced VEGF via inhibiting the AKT signaling pathway}

We found that Salmonella decreased VEGF expression in tumor cells by reducing AKT phosphorylation. The AKT/mTOR/p70S6K signaling pathway was reversed by transfecting a plasmid bearing a constitutively active form of AKT. The suppressive effect of Salmonella on the AKT/mTOR/p70S6K signaling pathway was relieved by transfecting a constitutively active form of AKT in B16F10 (Figure 3A) and 4T1 (Figure 3B) cells. Transfection of a plasmid encoding constitutively active AKT slightly increased the expression of HIF- $1 \alpha$ and VEGF by Salmonella treatment in comparison with the control group. The HIF-1 transcriptional activity was also rescued after Salmonella treatment by transfecting a plasmid bearing a constitutively active form of AKT (Figure 3C and 3D). There was a decrease in the proliferation of endothelial cells treated with conditioned medium from tumor cells infected with Salmonella. The phenomenon was reversed after transfecting a plasmid encoding constitutively active AKT in two tumor cell lines (Figure 4). Our results suggest that downregulation of AKT is required for the reduction of VEGF expression in tumor cells treated with Salmonella.

A

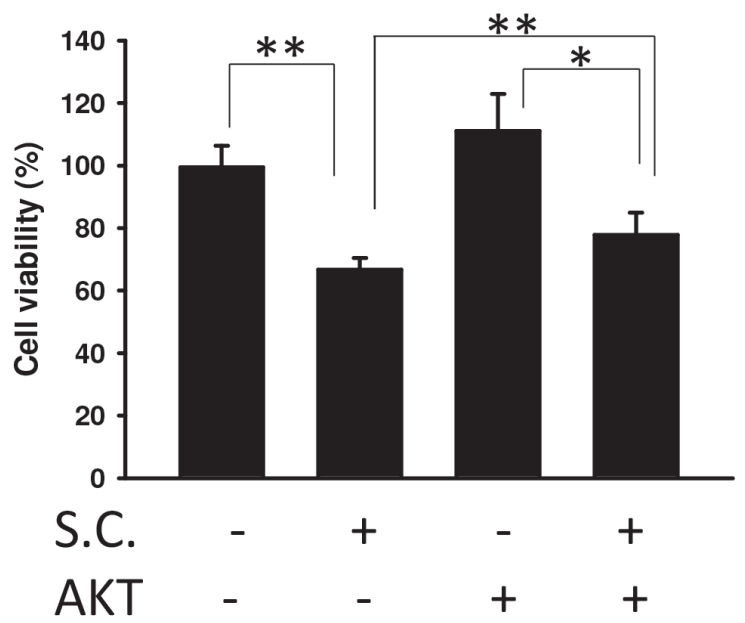

\section{Salmonella inhibited tumor growth in vivo}

The antitumor effects of Salmonella were evaluated in terms of tumor growth in mice bearing B16F10 or 4T1 tumors. As shown in Figure 5A and 5D, tumor growth was significantly retarded in mice treated with Salmonella in comparison with PBS-treated control mice. Figure 5B and $5 \mathrm{E}$ demonstrate that survival of the mice injected with Salmonella was significantly prolonged compared with mice injected with PBS. To investigate anti-angiogenesis in vivo after Salmonella treatment, mice bearing tumors were injected with Salmonella, and the levels of VEGF, the microvessel density, and the number of apoptotic cells in the tumors were determined by enzyme-linked immunosorbent assay (ELISA), immunohistochemistry, and terminal dUTP nick-end labeling (TUNEL), respectively (Figure 5C and 5F, and Figure 6). Salmonella reduced the levels of VEGF in tumor tissue homogenates (Figure 5C and 5F). Microvessel density within tumors from tumor-bearing mice was analyzed 7 days after Salmonella treatment by immunohistochemistry. The results of immunohistochemical staining are shown in Figure 6A. Tumors from Salmonella-treated mice appeared much less vascularized than their control counterparts (Figure 6C). TUNEL assays showed an increase in the amount of cells undergoing apoptosis in the Salmonellatreated tumors compared with PBS-treated tumors (Figure $6 \mathrm{~B})$. There was a 5.576.62-fold increase in the number of apoptotic cells induced by Salmonella compared with PBS-treated tumors (Figure 6D). Taken together, these results indicate that systemic delivery of Salmonella can delay tumor growth by reducing angiogenesis in tumors and enhance tumor cell death.

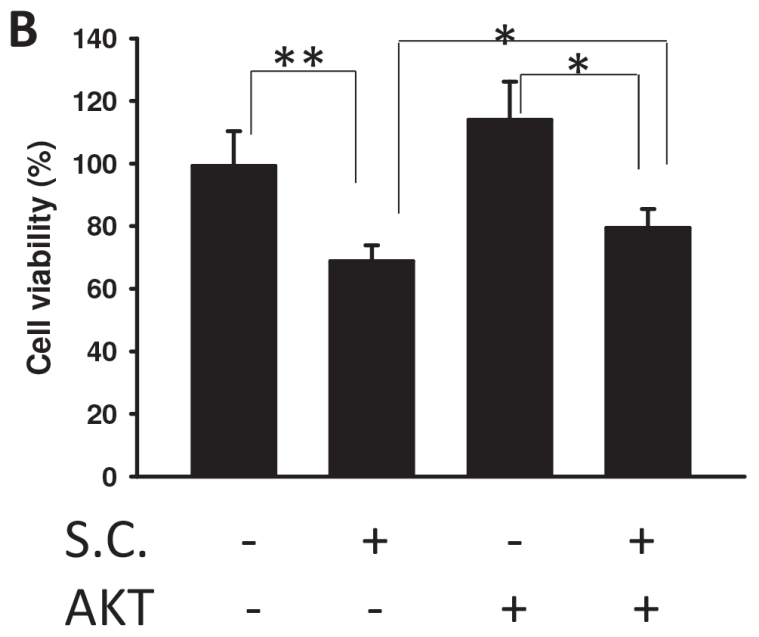

Figure 4: The effect of Salmonella on AKT phosphorylation and endothelial cell proliferation. The B16F10 A. and 4T1 B. cells transfected with control or constitutively active AKT plasmids were treated with Salmonella (MOI=10). The conditioned medium of those cells reduced the proliferation of endothelial cells. The HEMC-1 cells treated with conditioned medium from Salmonella-treated tumor cells were examined using the proliferation activity. Cell viability was assessed by the WST-1 assay. The data shown are the mean \pm $\mathrm{SD}(n=4)$. Multiplicity of infection (MOI). ${ }^{*} p<0.05 ; * * p<0.01$. 
A

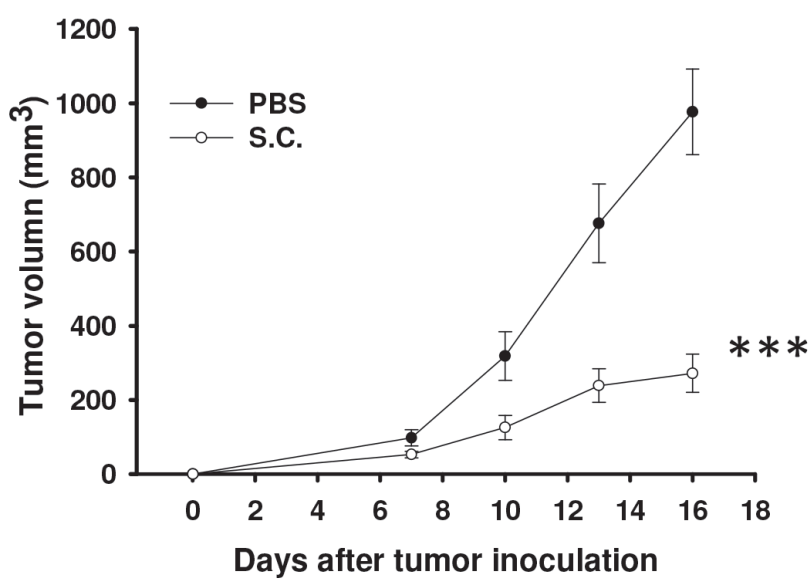

D

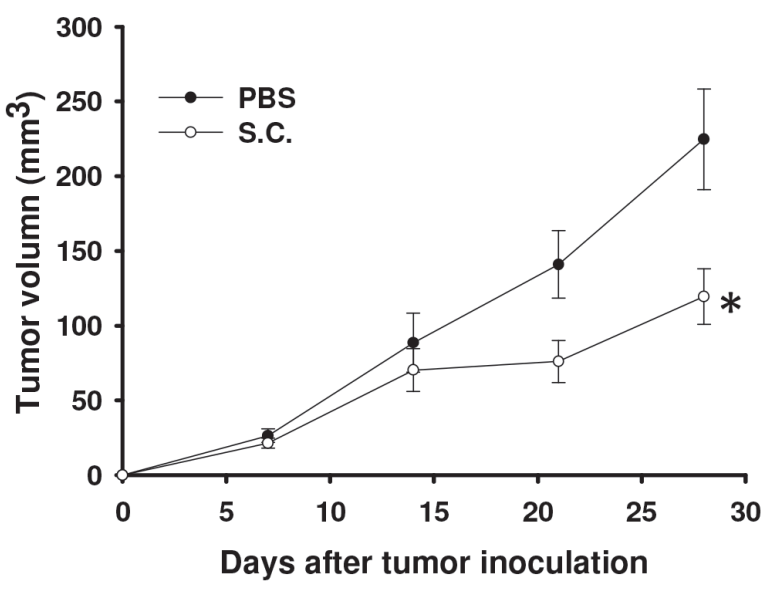

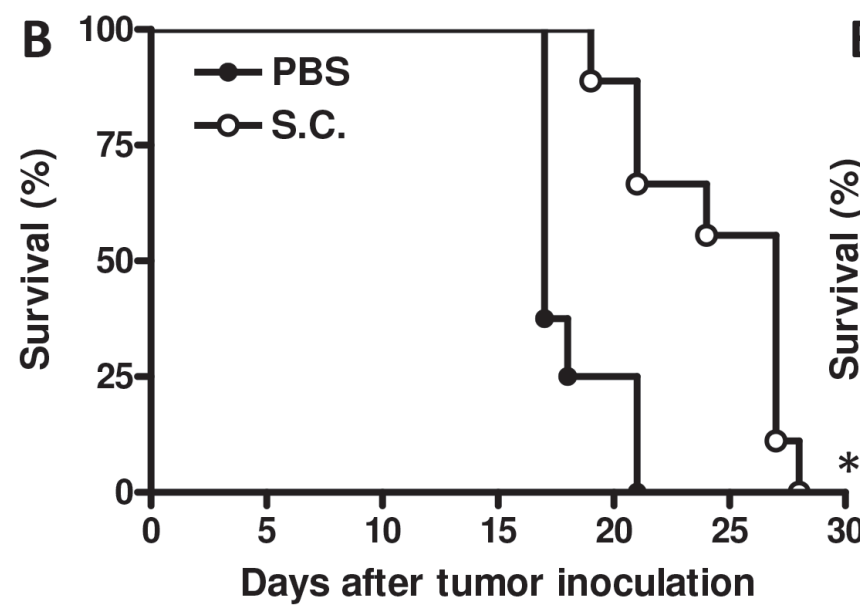

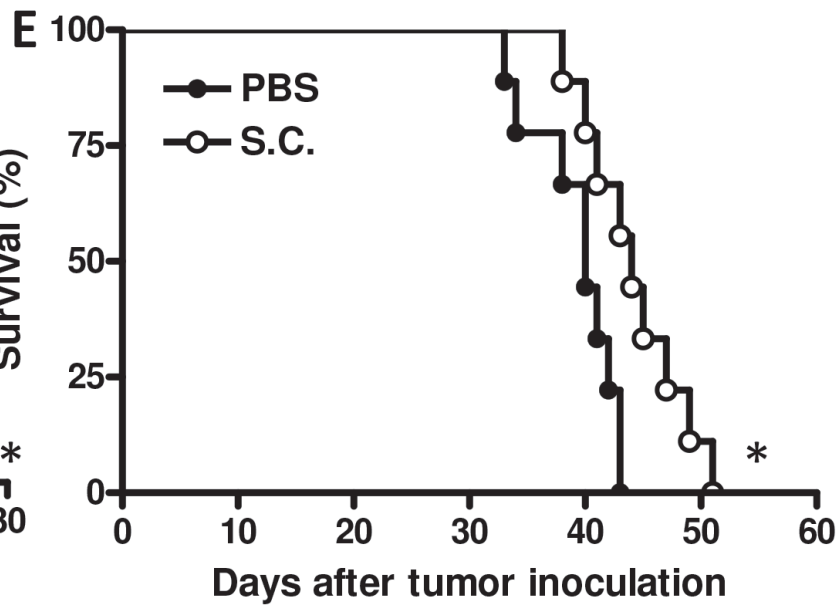

C

$\mathbf{F}$
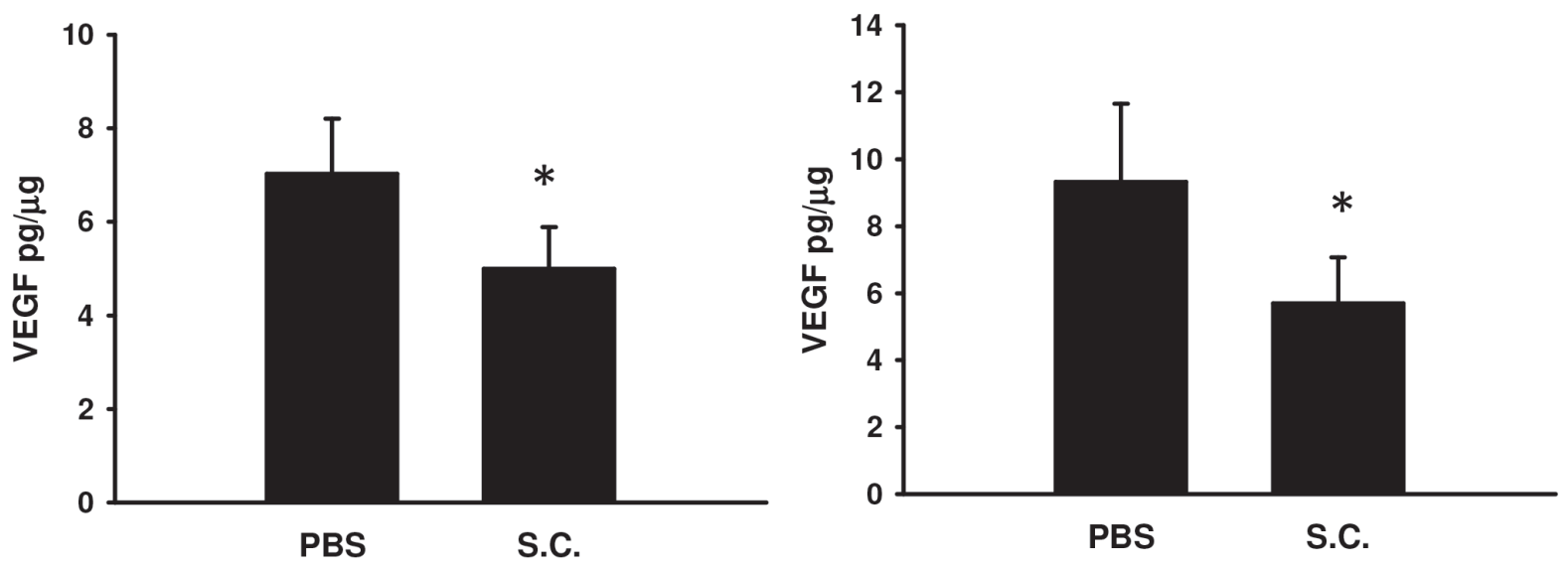

Figure 5: Salmonella (S.C.) retarded tumor growth and enhanced the survival of tumor-bearing mice. Groups of mice that had been inoculated subcutaneously with B16F10 and 4T1 at day 0 were treated i.p. with Salmonella $\left(10^{6} \mathrm{CFU}\right)$ at day 8 . Vehicle control mice were injected with PBS. The B16F10 A. and 4T1 D. tumor volumes were measured every 3 or 7 days after injection of Salmonella $(n=$ $8-9$, the data are the mean \pm SEM. $\left.{ }^{*} p<0.05 ; * * *<0.001\right)$. Kaplan-Meier survival curves of mice bearing B. B16F10 melanomas and E. 4T1 tumors exposed to various treatments are shown. The effect of Salmonella treatment on VEGF levels in tumor tissue homogenates. Groups of mice that had been inoculated subcutaneously with B16F10 and 4T1 at day 0 were treated i.p. with Salmonella (10 ${ }^{6}$ CFU) at day 8. Vehicle control mice were injected with PBS. The VEGF levels in B16F10 C. and 4T1 (F) tumors were measured by ELISA at day 15 after injection of Salmonella $(n=4$, data are mean $\pm \mathrm{SD}) .{ }^{*} p<0.05 ; * * * p<0.001$. 
A

B16F10

4T1
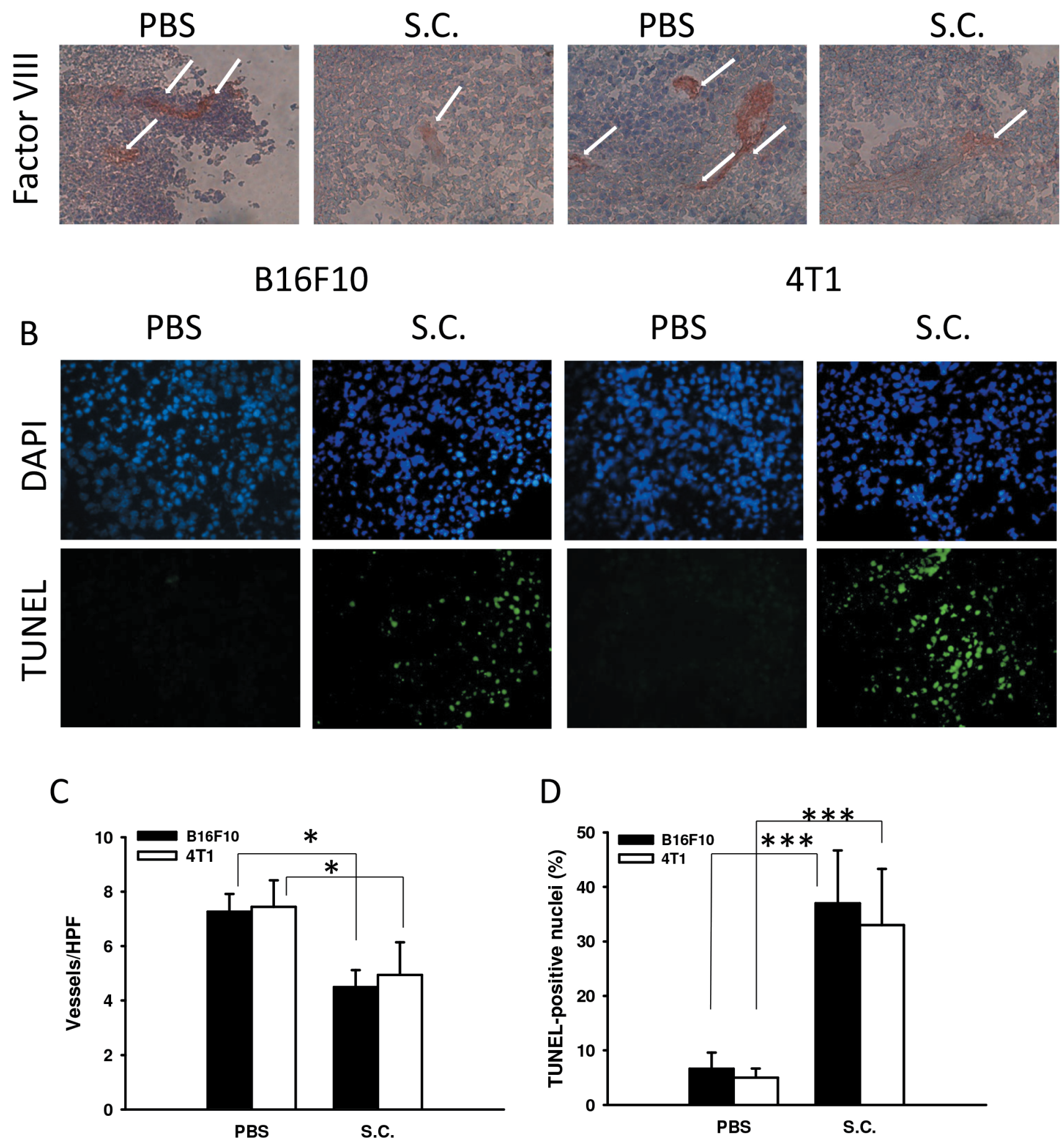

4T1

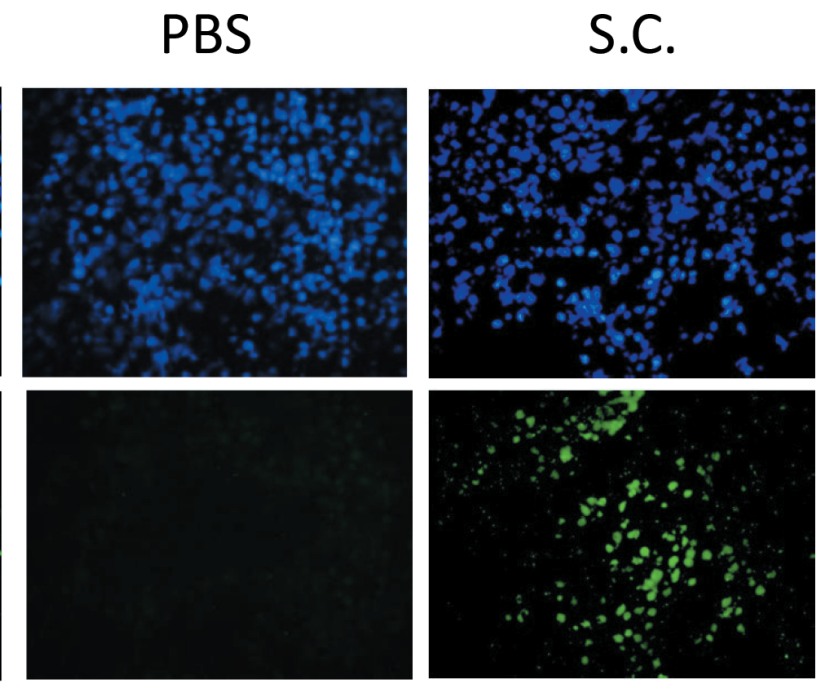

D

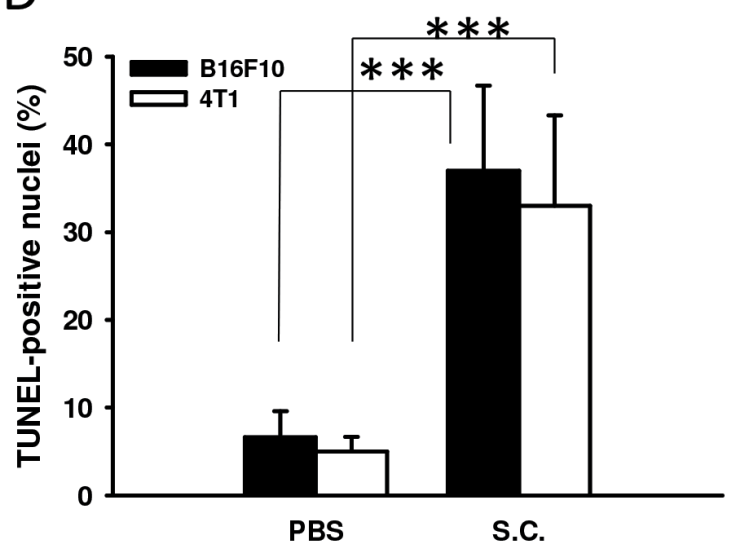

Figure 6: Salmonella reduced vessel density and enhanced tumor cell apoptosis. A. Tumors were excised at day 14, snap frozen and immunostained with rabbit antibody against factor VIII-related antigen $(\times 400)$. B. Tumors were excised at day 14 , and a TUNEL assay was used to detect apoptotic cells $(\times 400)$. C. Intratumoral microvessel density was determined by averaging the number of vessels in three areas of highest vessel density at $\times 400$ magnification in each section $(n=3$, the data are the mean $\pm \mathrm{SD}$. * $p<0.05)$. D. TUNEL-positive cells were counted from three fields of high-density positive cells in each section to determine the percentage of apoptotic cells $(n=3$, the data arethe mean $\pm \mathrm{SD} . * * * p<0.001)$. 


\section{DISCUSSION}

Salmonella may inhibit the proliferation and migration of tumor cells. Indeed, it has been demonstrated that VEGF directly stimulates the growth of tumor cells $[20,21]$. Salmonella inhibited the proliferation of endothelial cells and reduced the production of paracrine factors such as VEGF, thus suppressing the proliferation of tumor cells. Salmonella suppressed the expression of VEGF in tumor and angiogenic signaling cascades induced by HIF-1 $\alpha$. The anti-angiogenic effect of Salmonella may be similar to anti-VEGF antibody treatment, pruning immature vessels in tumor sites. The vessel normalization and restoration of pressure gradients induced by VEGF blockade may explain the increased replication of Salmonella in tumor sites and enhance the antitumor activities of Salmonella [15]. Anti-angiogenic agents may contribute to improving the hypoxic condition of tumor sites by vascular normalization $[6,22]$. Hypoxia, a hallmark of many solid tumors, was reduced by angiogenic inhibitors [23]. Herein, we showed that Salmonella had the ability to reduce HIF-1 $\alpha$ expression and may improve the hypoxic condition in the tumor microenvironment as well as increase the effects of radiation or chemotherapy. Indeed, the capability of Salmonella to disperse within tumors, and hence to reduce tumor growth, was augmented when combined with chemotherapy [15]. Several activities of Salmonella contribute to its antitumor effects. Salmonella has the ability to stimulate host immunity and increase tumor suppressor gene expression such as connexin 43 (Cx43) [24-26]. Regulation of $C x 43$ activity by Salmonella probably contributes to the anti-angiogenic activity [17]. Therefore, it is plausible that the antitumor effect of Salmonella may be attributed not only to its effects on tumor endothelial cells but also to its ability to enhance inflammation within tumor sites [24]. In addition, Western blot analysis showed that the expression levels of phospho-AKT (P-AKT), phospho-mTOR (P-mTOR), and phospho-p70s6K(P-p70s6K) in tumor cells were decreased after Salmonella infection. Activation of AKT is one of the major mechanisms of tumorigenesis, and blocking this signaling pathway could have therapeutic implications for tumors. Previous studies demonstrated that the activation of AKT is associated with intrinsic radioresistance, tumor cell proliferation, and angiogenesis in vivo [27]. AKT can affect proliferation signaling and induce anti-apoptotic effects in tumors. Transgenic mice expressing active AKT develop thymoma and mammary tumors [27, 28]. Our results indicate that Salmonella inhibits HIF-1 $\alpha$ expression via downregulation of the AKT/mTOR pathway. Herein, we demonstrate that Salmonella-mediated AKT/mTOR downregulation is an important modulator of HIF- $1 \alpha$ expression and plays a crucial role in antitumor therapy.

Salmonella had demonstrated immunopotentiating properties [29] and anti-angiogenic activity [30]. The induction of tumor necrosis factor- $\alpha$ in host cells acts as a vascular disrupting agent after Salmonella treatment [31]. Meanwhile, Salmonella inhibits the expression of VEGF in tumor-associated macrophages [32]. However, the successful induction of antitumor effects in subcutaneous tumors may not necessarily indicate efficacy against orthotopic tumors. Therefore, orthotopic tumor models are preferred for analyzing the tumoricidal effect of Salmonella [33, 34]. Our previous results revealed that Salmonella accumulated in not only subcutaneous but also orthotopic tumors after systemic administration [35]. Salmonella may be beneficial to cancer treatment in the future.

\section{MATERIALS AND METHODS}

\section{Bacteria, cell lines, plasmids and mice}

A vaccine strain of Salmonella enterica serovar choleraesuis (S .choleraesuis) (ATCC 15480) was obtained from the Bioresources Collection and Research Center (Hsinchu, Taiwan). This rough variant of S. choleraesuis (S.C.) was designated vaccine 51 [26]. Murine melanoma B16F10 cells [36] and murine breast cancer 4T1 cells [37] were cultured in Dulbecco's modified Eagle's medium (DMEM) supplemented with $50 \mu \mathrm{g} / \mathrm{ml}$ gentamicin, 2 $\mathrm{mM} \mathrm{L}$-glutamine, and $10 \%$ heat-inactivated fetal bovine serum (FBS) at $37^{\circ} \mathrm{C}$ in $5 \% \mathrm{CO}_{2}$. Human HMEC-1 microvascular endothelial cells $[14,17]$ were cultured in EGM endothelial growth medium (Cambrex, East Rutherford, NJ, USA). Constitutively active AKT plasmid was kindly provided by Dr. Chiau-Yuang Tsai (Department of Molecular Immunology, Osaka University) [38]. The 24-bp HRE (5'-CAC ACG TGG GTT CCC GCA CGT CCG-3') of the human lactic dehydrogenase A gene was obtained by polymerase chain reaction, and 6 copies of this fragment were individually tandemly ligated into the 5' region of the CMV minimal (CMVmini) promoter derived from the pTRE vector (Clontech, Palo Alto, CA) at the $S t u \mathrm{I} / E c o$ RI sites. To construct luciferase reporter plasmids, six copies of HRE ligated to the CMV minimal promoter $(6 \times \mathrm{HRE} / \mathrm{CMVmini})$ were excised from the pTRE-based plasmids by digestion with KpnI and HindIII and subcloned into pGL3 (Promega, Madison, WI) at the $K p n \mathrm{I} / H i n d I I I$ sites. To construct the lentiviral vector, the fragment of $6 \times \mathrm{HRE} / \mathrm{CMVmini}-\mathrm{Luc}$ was excised from pGL3 by digestion with $K p n \mathrm{I} / \mathrm{Bg} / \mathrm{II}$ and subcloned into pMECA. The fragment of $6 \times \mathrm{HRE} / \mathrm{CMVmini}-\mathrm{Luc}$ was released from the pMECA-based plasmid by digestion with $\mathrm{ClaI} / \mathrm{SwaI}$ sites and cloned into pWPXL [16]. Six-toeight-week-old female BABL/c and C57BL/6 mice were obtained from the National Laboratory Animal Center of Taiwan. The animals were maintained in a specialized pathogen-free animal care facility in isothermal conditions with regular photoperiods. The experimental protocol 
adhered to the rules of the Animal Protection Act of Taiwan and was approved by the Laboratory Animal Care and Use Committee of the China Medical University (permit number: 104-24-N).

\section{Endothelial cell viability assay}

HMEC- 1 cells $\left(2 \times 10^{3} /\right.$ well $)$ were cultured in 96well plates. Tumor cells $\left(10^{5} /\right.$ well $)$ that had been cultured in 6-well plates overnight were infected with various MOIs of Salmonella or mock-infected with antibiotic-free culture medium for $4 \mathrm{~h}$. The medium was then removed, and cells were washed and replenished with fresh medium supplemented with $2 \%$ FBS and $50 \mu \mathrm{g} / \mathrm{ml}$ gentamicin. After $48 \mathrm{~h}$, the conditioned medium was collected, filtered through a $0.22-\mu \mathrm{m}$ filter, and analyzed for its ability to inhibit endothelial cell proliferation. The culture HMEC-1 medium was then removed and replaced with conditioned medium. After $48 \mathrm{~h}$, cell proliferation was assessed by the colorimetric WST-1 assay (Dojindo Labs, Tokyo, Japan) according to the manufacturer's instructions [38].

\section{Analysis of hypoxia-inducible transcriptional activities}

Various cells grown in 24-well plates were cotransfected with luciferase reporter plasmids driven by HRE promoters $(0.66 \mu \mathrm{g})$ and pTCYLacZ $(0.34 \mu \mathrm{g})$, a $\beta$-galactosidase $(\beta$-gal) expression plasmid driven by the $\beta$-actin promoter, using Lipofectamine 2000. The culture medium was then removed and replaced with conditioned medium. At post-transfection, cell lysates were harvested $16 \mathrm{~h}$ later. The cell lysates were assessed for their luciferase activities, as determined by a dual-light luciferase and $\beta$-gal reporter gene assay system (Applied Biosystems, Foster City, CA, USA) using a luminometer (Minilumate LB9506, Bad Wildbad, Germany). Relative luciferase activity was measured as luciferase activity divided by $\beta$-gal activity to normalize transfection efficiency per microgram protein. The protein content in each sample was determined by the bicinchoninic acid (BCA) protein assay (Pierce Biotechnology, Rockford, IL). The cells with high levels of luciferase expression were transfected with control or constitutively active AKT plasmids. The luciferase activity was assessed by a luminometer as previously described.

\section{Animal studies}

Groups of mice were subcutaneously (s.c.) inoculated with 106 tumor cells. When the tumors had grown to diameters between 50 and $100 \mathrm{~mm}^{3}$, the mice were intraperitoneally (i.p.) injected with $2 \times 10^{6}$ colonyforming units (cfu) of Salmonella. Palpable tumors were measured every 3 days or 7 days along two perpendicular axes using a tissue caliper, and the tumor volumes were calculated as follows: (length of tumor) $\times$ (width of the tumor) ${ }^{2} \times 0.45$. All mice were monitored for tumor growth and survival as previously described [14].

\section{Immunoblot analysis}

The protein content in each sample was determined using a BCA protein assay (Pierce Biotechnology). Proteins were fractionated by SDS-PAGE, transferred onto Hybond enhanced chemiluminescence nitrocellulose membranes (Amersham, Little Chalfont), and probed with antibodies against HIF-1 $\alpha$ (Novus Biologicals, Littleton, $\mathrm{CO}$ ), VEGF (Novus Biologicals), the mammalian target of rapamycin (mTOR) (Cell Signaling, Danvers, MA, USA), phospho-mTOR (Cell Signaling), AKT (Santa Cruz Biotechnology, Inc. Santa Cruz, CA, USA), phospho-AKT (Santa Cruz Biotechnology, Inc.), p70 S6 kinase (p70S6K) (Cell Signaling), phospho-p70S6K (Cell Signaling), or monoclonal antibodies against $\beta$-actin (AC-15, Sigma Aldrich). Horseradish peroxidase-conjugated goat antimouse IgG or anti-rabbit IgG (Jackson, West Grove, PA, USA) was used as the secondary antibody, and proteinantibody complexes were visualized by an enhanced chemiluminescence system (Amersham). The signals were quantified with ImageJ software (rsbweb.nih.gov/ij/) [39].

\section{ELISA, immunohistochemistry and TUNEL}

The levels of mouse VEGF in tumor tissue homogenates were determined by ELISA [17]. To analyze microvessel density in the tumor sites, the whole tumors were excised and snap frozen at day 14. Frozen tumor sections were prepared according to the aforementioned procedure, and incubated with rabbit anti-factor VIIIrelated antigen (DAKO, Carpinteria, CA). After sequential incubation with appropriate peroxidase-labeled secondary antibody and aminoethyl carbazole (AEC) as the substrate chromogen, tumor sections were counterstained with hematoxylin. Areas containing the highest number of capillaries were identified by scanning the tumor sections at $\times 100$ magnification. After the fields of high microvessel density (neovascular "hot spots") were determined, individual vessels were counted in $\times 400$ magnifications. Microvessel density was determined by averaging the number of microvessels in the three areas of highest vessel density at $\times 400$ magnification in each section. The orientation of the sections was random [14,17]. A TUNEL assay was used to detect cell death in tumors and was performed according to the manufacturer's instructions (Promega, Madison, WI). We used three high-power $(\times$ 400) fields with approximately 200-300 cells that showed the highest density of positive-stained cells per field to determine the average percentage of apoptotic (TUNEL 
positive) cells in each section [35].

\section{Statistical analysis}

The unpaired, two-tailed Student's t test was used to determine differences between groups for comparison with the control group. A survival analysis was performed using the Kaplan-Meier survival curve and log-rank test. A $p$ value less than 0.05 was considered to be statistically significant.

\section{ACKNOWLEDGMENTS}

This work was supported by the Ministry of Science and Technology, Taiwan (MOST 104-2320-B039-042MY3), China Medical University (CMU-104-S-18) and Distmanson Medical Foundation Chia-Yi Christian Hospital Research Program (R104-031).

\section{CONFLICTS OF INTEREST}

The authors declare no conflict of interest.

\section{REFERENCES}

1. Hiroshima Y, Zhao M, Zhang Y, Zhang N, Maawy A, Murakami T, Mii S, Uehara F, Yamamoto M, Miwa S, Yano S, Momiyama M, Mori R, Matsuyama R, Chishima T, Tanaka K, Ichikawa Y, Bouvet M, Endo I, Hoffman RM. Tumor-targeting Salmonella typhimurium A1-R arrests a chemo-resistant patient soft-tissue sarcoma in nude mice. PLoS One 2015;10: e0134324.

2. Matsumoto Y, Miwa S, Zhang Y, Zhao M, Yano S, Uehara F, Yamamoto M, Hiroshima Y, Toneri M, Bouvet M, Matsubara H, Tsuchiya H, Hoffman RM. Intraperitoneal administration of tumor-targeting Salmonella typhimurium A1-R inhibits disseminated human ovarian cancer and extends survival in nude mice. Oncotarget 2015;6: 1136911377. doi: 10.18632/oncotarget.3607.

3. Zhang Y, Zhang N, Zhao M, Hoffman RM. Comparison of the selective targeting efficacy of Salmonella typhimurium A1-R and VNP20009 on the Lewis lung carcinoma in nude mice. Oncotarget. 2015;6: 14625-14631. doi 10.18632/ oncotarget.3342.

4. Zhang Y, Miwa S, Zhang N, Hoffman RM, Zhao M. Tumor-targeting Salmonella typhimurium A1-R arrests growth of breast-cancer brain metastasis. Oncotarget. 2015;6: 2615-2622. doi: 10.18632/oncotarget.2811.

5. Miwa S, Zhang Y, Baek KE, Uehara F, Yano S, Yamamoto M, Hiroshima Y, Matsumoto Y, Kimura H, Hayashi K, Yamamoto N, Bouvet M, Tsuchiya H, Hoffman RM, Zhao M. Inhibition of spontaneous and experimental lung metastasis of soft-tissue sarcoma by tumor-targeting Salmonella typhimurium A1-R. Oncotarget. 2014;5: 12849-
12861. doi: 10.18632/oncotarget.2561.

6. Hiroshima Y, Zhang Y, Murakami T, Maawy A, Miwa S, Yamamoto M, Yano S, Sato S, Momiyama M, Mori R, Matsuyama R, Chishima T, Tanaka K, Ichikawa Y, Bouvet M, Endo I, Zhao M, Hoffman RM. Efficacy of tumortargeting Salmonella typhimurium A1-R in combination with anti-angiogenesis therapy on a pancreatic cancer patient-derived orthotopic xenograft (PDOX) and cell line mouse models. Oncotarget 2014;5: 12346-12357. doi: 10.18632/oncotarget.2641.

7. Miwa S, Yano S, Zhang Y, Matsumoto Y, Uehara F, Yamamoto M, Hiroshima Y, Kimura H, Hayashi K, Yamamoto N, Bouvet M, Tsuchiya H, Hoffman RM, Zhao M. Tumor-targeting Salmonella typhimurium A1-R prevents experimental human breast cancer bone metastasis in nude mice. Oncotarget 2014;5: 7119-7125. doi: 10.18632/oncotarget.2226.

8. Matsumoto Y, Miwa S, Zhang Y, Hiroshima Y, Yano S, Uehara F, Yamamoto M, Toneri M, Bouvet M, Matsubara H, Hoffman RM, Zhao M. Efficacy of tumor-targeting Salmonella typhimurium A1-R on nude mouse models of metastatic and disseminated human ovarian cancer. J Cell Biochem 2014;115: 1996-2003.

9. Hiroshima Y, Zhao M, Zhang Y, Maawy A, Hassanein MK, Uehara F, Miwa S, Yano S, Momiyama M, Suetsugu A, Chishima T, Tanaka K, Bouvet M, Endo I, Hoffman RM. Comparison of efficacy of Salmonella typhimurium A1-R and chemotherapy on stem-like and non-stem human pancreatic cancer cells. Cell Cycle 2013;12: 2774-2780.

10. Chang WW, Lee CH. Salmonella as an innovative therapeutic antitumor agent. Int J Mol Sci 2014; 15:1454614554.

11. Lee $\mathrm{CH}$. Engineering bacteria toward tumor targeting for cancer treatment: current state and perspectives. Appl Microbiol Biotechnol 2012;93: 517-523.

12. Lee $\mathrm{CH}$, Hsieh JL, Wu CL, Hsu PY, Shiau AL. T cell augments the antitumor activity of tumor-targeting Salmonella. Appl Microbiol Biotechnol 2011;90: 13811388.

13. Lee $\mathrm{CH}, \mathrm{Wu} \mathrm{CL}$, Shiau AL. Toll-like receptor 4 mediates an antitumor host response induced by Salmonella choleraesuis. Clin Cancer Res 2008;14: 1905-1912.

14. Lee $\mathrm{CH}, \mathrm{Wu} \mathrm{CL}$, Shiau AL. Endostatin gene therapy delivered by Salmonella choleraesuis in murine tumor models. J Gene Med 2004;6: 1382-1393.

15. Lee $\mathrm{CH}$, Wu CL, Tai YS, Shiau AL. Systemic administration of attenuated Salmonella choleraesuis in combination with cisplatin for cancer therapy. Mol Ther 2005;11: 707-716.

16. Lee $\mathrm{CH}, \mathrm{Wu} \mathrm{CL}$, Shiau AL. Hypoxia-induced cytosine deaminase gene expression for cancer therapy. Hum Gene Ther 2007;18: 27-38.

17. Wang WK, Chen MC, Leong HF, Kuo YL, Kuo CY, Lee $\mathrm{CH}$. Connexin 43 suppresses tumor angiogenesis by 
down-regulation of vascular endothelial growth factor via hypoxic-induced factor-1 $\alpha$. Int J Mol Sci 2015;16: 439-451.

18. Chen MC, Hsu WL, Hwang PA, Chou TC. Low molecular weight fucoidan inhibits tumor angiogenesis through downregulation of HIF-1/VEGF signaling under Hypoxia. Mar Drugs 2015;13: 4436-4451.

19. Ataie-Kachoie P, Pourgholami MH, Bahrami-B F, Badar $\mathrm{S}$, Morris DL. Minocycline attenuates hypoxia-inducible factor-1 $\alpha$ expression correlated with modulation of $\mathrm{p} 53$ and AKT/mTOR/p70S6K/4E-BP1 pathway in ovarian cancer: in vitro and in vivo studies. Am J Cancer Res 2015;5: 575588.

20. Tse LY, Sun X, Jiang H, Dong X, Fung PW, Farzaneh $\mathrm{F}, \mathrm{Xu} \mathrm{R}$. Adeno-associated virus-mediated expression of kallistatin suppresses local and remote hepatocellular carcinomas. J Gene Med 2008;10: 508-517.

21. Tong RT, Boucher Y, Kozin SV, Winkler F, Hicklin DJ, Jain RK. Vascular normalization by vascular endothelial growth factor receptor 2 blockade induces a pressure gradient across the vasculature and improves drug penetration in tumors. Cancer Res 2004;64: 3731-3736.

22. Lee CG, Heijn M, di Tomaso E, Griffon-Etienne G, Ancukiewicz M, Koike C, Park KR, Ferrara N, Jain RK, Suit HD, Boucher Y. Anti-Vascular endothelial growth factor treatment augments tumor radiation response under normoxic or hypoxic conditions. Cancer Res 2000;60: 5565-5570.

23. Teicher BA, Holden SA, Ara G, Dupuis NP, Liu F, Yuan J, Ikebe M, Kakeji Y. Influence of an anti-angiogenic treatment on 9L gliosarcoma: oxygenation and response to cytotoxic therapy. Int J Cancer 1995;61: 732-737.

24. Saccheri F, Pozzi C, Avogadri F, Barozzi S, Faretta M, Fusi $\mathrm{P}$, Rescigno M. Bacteria-induced gap junctions in tumors favor antigen cross-presentation and antitumor immunity. Sci Transl Med 2010;2: 44ra57.

25. Chang WW, Lai CH, Chen MC, Liu CF, Kuan YD, Lin $\mathrm{ST}$, Lee CH. Salmonella enhance chemosensitivity in tumor through connexin 43 upregulation. Int J Cancer 2013 15;133: 1926-1935.

26. Wang WK, Kuan YD, Kuo CY, Lee CH. Connexin 43 gene therapy delivered by polymer-modified Salmonella in murine tumor models. Polymers 2014; 6:1119-1128.

27. Moral M, Segrelles C, Lara MF, Martínez-Cruz AB, Lorz C, Santos M, García-Escudero R, Lu J, Kiguchi K, Buitrago A, Costa C, Saiz C, Rodriguez-Peralto JL, Martinez-Tello FJ. et al. Akt activation synergizes with Trp53 loss in oral epithelium to produce a novel mouse model for head and neck squamous cell carcinoma. Cancer Res 2009;69: 10991108.

28. Blanco-Aparicio C, Pérez-Gallego L, Pequeño B, Leal JF, Renner O, Carnero A. Mice expressing myrAKT1 in the mammary gland develop carcinogen-induced ERpositive mammary tumors that mimic human breast cancer. Carcinogenesis 2007;28: 584-594.
29. Hong EH, Chang SY, Lee BR, Pyun AR, Kim JW, Kweon $\mathrm{MN}$, Ko HJ. Intratumoral injection of attenuated Salmonella vaccine can induce tumor microenvironmental shift from immune suppressive to immunogenic. Vaccine 2013;31: 1377-1384.

30. al-Ramadi BK, Fernandez-Cabezudo MJ, El-Hasasna H, AlSalam S, Bashir G, Chouaib S. Potent anti-tumor activity of systemically-administered IL2-expressing Salmonella correlates with decreased angiogenesis and enhanced tumor apoptosis. Clin Immunol 2009;130: 89-97.

31. Leschner S, Westphal K, Dietrich N, Viegas N, Jablonska J, Lyszkiewicz M, Lienenklaus S, Falk W, Gekara N, Loessner H, Weiss S. Tumor invasion of Salmonella enterica serovar Typhimurium is accompanied by strong hemorrhage promoted by TNF- $\alpha$. PLoS One 2009;4: e6692.

32. Kaimala S, Mohamed YA, Nader N, Issac J, Elkord E, Chouaib S, Fernandez-Cabezudo MJ, Al-Ramadi BK. Salmonella-mediated tumor regression involves targeting of tumor myeloid suppressor cells causing a shift to M1-like phenotype and reduction in suppressive capacity. Cancer Immunol Immunother 2014;63: 587-599.

33. Hoffman RM. Orthotopic metastatic mouse models for anticancer drug discovery and evaluation: a bridge to the clinic. Invest New Drugs 1999;17: 343-359.

34. Hoffman RM. Patient-derived orthotopic xenografts: better mimic of metastasis than subcutaneous xenografts. Nat Rev Cancer 2015;15: 451-452.

35. Lee $\mathrm{CH}, \mathrm{Wu} \mathrm{CL}$, Shiau AL.Salmonella choleraesuis as an anticancer agent in a syngeneic model of orthotopic hepatocellular carcinoma. Int J Cancer 2008;122:930-935.

36. Lee CH, Lin ST, Liu JJ, Chang WW, Hsieh JL, Wang WK.Salmonella induce autophagy in melanoma by the downregulation of AKT/mTOR pathway. Gene Ther 2014;21:309-316.

37. Chang WW, Kuan YD, Chen MC, Lin ST, Lee CH.Tracking of mouse breast cancer stem-like cells with Salmonella. Exp Biol Med 2012;237:1189-1196.

38. Shiau AL, Shen YT, Hsieh JL, Wu CL, Lee CH.Scutellaria barbata inhibits angiogenesis through downregulation of HIF-1 $\alpha$ in lung tumor. Environ Toxicol 2014;29:363-370.

39. Chang WW, Liu JJ, Liu CF, Liu WS, Lim YP, Cheng YJ, Lee CH.An extract of Rhodobacter sphaeroides reduces cisplatin-induced nephrotoxicity in mice.Toxins 2013;5:2353-2365. 